\title{
Constraints on the Use of de Wit Models to Analyze Competitive Interactions
}

\author{
M. R. Newton, L. L. Kinkel, and K. J. Leonard
}

First, second, and third authors: Department of Plant Pathology, University of Minnesota, 495 Borlaug Hall, 1991 Upper Buford Circle, St. Paul; and third author: USDA, ARS Cereal Disease Laboratory, University of Minnesota. Accepted for publication 9 June 1998.

The de Wit (5) replacement series is one of the most widely used designs for the study of competitive interactions in the ecological literature (2) and has been used in several recent studies of microbial interactions $(1,4,14,17-19)$. In replacement series studies, the proportions of two microbial strains in a mixture are varied, while the total population density of the mixture is held constant, and the relative yields (RYs) of two strains as well as the relative yield total (RYT) for the two are analyzed as functions of the varying proportions of the strains. RY is the reproductive output of a strain in the mixture relative to its output alone at the same total population density, and RYT is the sum of the RYs of the two strains.

The null hypothesis in the traditional interpretation of de Wit curves is that inter- and intrastrain competitive abilities are equal, so the expected RY of each strain in the mixture is equal to its proportion in the mixture. Thus, in the null hypothesis, the expected relationship between strain frequency and RY in the mixture is linear, and the expected RYT is 1.0 over all strain frequencies (Fig. 1). If a curve for a strain's observed RY plotted against its frequency in mixture lies above the null straight line, the traditional interpretation is that this strain's reproductive output per individual is more strongly inhibited by other individuals of the same strain than by individuals of the other strain. Conversely, a RY curve that falls below the null straight line is generally interpreted as indicating that this strain is more strongly inhibited by the other strain than by itself.

There have been several criticisms of de Wit analysis. One is that typical de Wit replacement series involves only one total density, and, therefore, does not take into account the expected density dependence of competition $(6,16)$. Also, in replacement series, effects of changes in the density of one strain are confounded by effects of changes in the density of the other, making it impossible to fully describe reproductive dynamics in mixture as a function of the densities of both strains (16). Recently, Newton (10) identified another limitation in using de Wit models to quantify competitive interactions. de Wit curves constructed for pathogens, or any organisms whose life cycles have multiple stages at which competitive interactions may occur, may not correspond to the traditional expectations for particular combinations of intra- and interstrain interactions in a system. Specifically, the RY curves may deviate from the null lines as a result of differences in ecological traits other than competitive ability (e.g., carrying capacity, infection efficiency) among organisms in mixed populations, or they may devi-

Corresponding author: M. R. Newton

E-mail address: miriamn@puccini.crl.umn.edu

Publication no. P-1998-0707-010

This article is in the public domain and not copyrightable. It may be freely reprinted with customary crediting of the source. The American Phytopathological Society, 1998. ate from the null lines in unexpected patterns when competitive differences do exist.

\section{MODELS OF DENSITY-DEPENDENT INTERACTION OF STRAINS}

We constructed de Wit curves using output from two models that we developed to describe competitive interactions between coexisting strains of plant pathogens. We originally developed these models for the system of wheat stem rust (Puccinia graminis $\mathrm{f}$. sp. tritici) on wheat leaves (10-13), but they have also been applied to epiphytic bacteria (9) and are generally applicable to other organisms that exhibit similar density-dependent patterns of reproduction $(12,13)$. The models represent relationships between population density and reproductive output in two distinct life cycle stages, colonization (e.g., for $P$. graminis f. sp. tritici $=$ infection) and reproduction (e.g., for $P$. graminis f. sp. tritici $=$ sporulation). A summary of the model derivations is presented below; details are given by Newton et al. $(12,13)$.

The colonization model shares fundamental assumptions with the well-known multiple-infection transformation (7): the number of infectable (colonizable) sites on leaves is finite and these sites may be infected by multiple inoculated propagules, but only one successful colony arising from one propagule can develop in a site. When only one strain is present on a leaf, the density-output relationship is described by

$$
U=K\left[1-\left(1-\frac{E}{K}\right)^{S}\right],
$$

in which $U=$ number of reproductively active colonies or sporulating lesions on a leaf; $K=$ carrying capacity (i.e., maximum number) of colonies on the leaf; $E=$ infection efficiency, or probability that a propagule, once inoculated, will infect the leaf; and $S=$ number of propagules inoculated onto the leaf. When two strains coexist on a leaf, the function is expanded to describe the output of one of the strains (strain 1) as

$$
U_{1}=K_{1} \sum_{x_{1}=1}^{S_{1}} \sum_{x_{2}=0}^{S_{2}}\left(\frac{x_{1}}{x_{1}+\alpha_{2} x_{2}}\right) \operatorname{Pr}\left(x_{1}\right) \operatorname{Pr}\left(x_{2}\right),
$$

in which $x_{\mathrm{i}}$ is the number of infections of strain $\mathrm{i}$ in a given infection site, $\operatorname{Pr}\left(x_{\mathrm{i}}\right)$ is a binomial $\left(S_{\mathrm{i}}, E_{\mathrm{i}} / K_{1}\right)$ probability $(8)$ that $x_{\mathrm{i}}$ infections of strain $i$ will form in the site, and $\alpha_{2}$ is the interstrain interaction effect of strain 2 on colony formation by strain 1 . Biologically, $\alpha_{2}$ represents the number of infections of strain 1 that would have the same inhibitory effect on colony formation by strain 1 as does a single infection of strain 2, within a given site. The output of strain 2 is obtained by reversing the subscripted $1 \mathrm{~s}$ and $2 \mathrm{~s}$ in the equation; the values of $K, E$, and $\alpha$ are potentially different for strains 1 and 2 . 
In the reproduction model, derived from a monomolecular function (3), the density-output relationship for a single strain on a leaf is

$$
Q=M\left[1-\left(1-\frac{A}{M}\right)^{U}\right],
$$

in which $Q=$ number of propagules produced by colonies on a leaf; $M=$ maximum propagule production on the leaf; $A=$ propagation efficiency, or intrinsic number of propagules produced per colony in the absence of intra- or interstrain interaction; and $U$ is as defined above. When two strains coexist on a leaf, equation 3 is modified as

$$
Q_{1}=\left(\frac{U_{1}}{U_{1}+\beta_{2} U_{2}}\right) M_{1}\left[1-\left(1-\frac{A_{1}}{M_{1}}\right)^{U_{1}+\beta_{2} U_{2}}\right],
$$

in which $\beta_{2}$ is the interstrain interaction effect of strain 2 on the reproductive output of strain 1 . Biologically, $\beta_{2}$ represents the number of colonies of strain 1 that would have the same inhibitory effect on propagule production by strain 1 as does a single colony of strain 2. Propagule production of each strain in mixture can ultimately be predicted as a function of the initial inoculum densities of the strains by coupling the two models.

Both models quantify analogous types of ecological traits affecting pathogen fitness (per-individual contribution to the next generation's gene pool) (15): carrying capacities for colonies or propagules produced; reproductive efficiencies, or intrinsic reproductive output per individual in the absence of interactions; and interaction coefficients, or the effects of a strain on the output of the other. The interaction coefficients in both models are general measures encompassing any type of inhibitory (competitive or antagonistic) or synergistic interaction: a strain with a coefficient value $>1$ inhibits the other strain's output more strongly than the other inhibits itself; a value of 1 indicates equal inter- and intrastrain com-

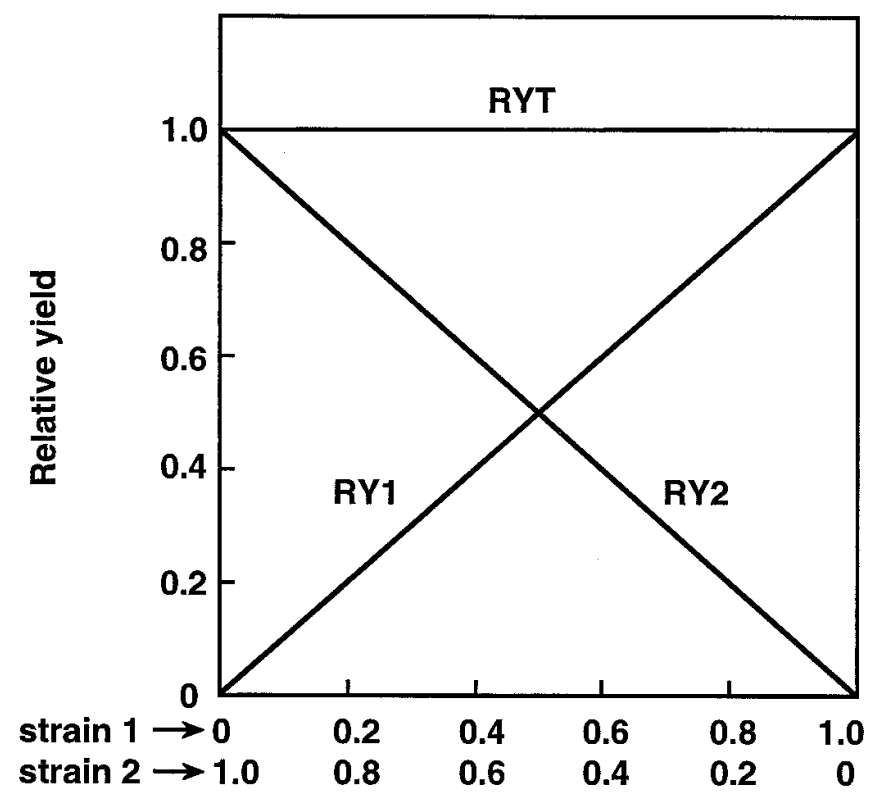

\section{Proportion of strains in inoculum}

Fig. 1. Expected (null) de Wit curves for the case of equal inter- and intrastrain competition for two coexisting pathogen strains. Strains have equal baseline parameter values (Table 2). Traditionally, it has been assumed that if inter- and intrastrain competition are equal for both strains, the relative yield (RY) of each strain (reproductive output of a strain in mixture relative to its output alone at the same total population density) will be a linear function of its frequency in mixture, and the relative yield total (RYT) for the two strains (sum of RYs of the two strains) will have a constant value of 1.0 at all strain frequencies. petition; a value between 0 and 1 indicates that the strain affects the other strain more weakly than the other affects itself; and a value $<0$ is indicative of a synergistic effect on the other strain. Both models also describe similar saturation curves, in which output increases with increasing density, but levels off asymptotically as it approaches the carrying capacity. The single-strain functions of the models are, in fact, identical; the difference in the mixedstrain functions reflects different assumptions about the nature of interactions in the two life cycle stages. In the colonization model, the multiple-infection transformation assumes that interactions among developing infections or colonies occur on a localized scale to capture sites to establish mature colonies, whereas the reproduction model assumes nonlocalized interaction of colonies across the leaf to capture leaf resources for reproduction (12).

\section{DE WIT CURVES GENERATED FROM INTERACTION MODELS}

Using the models, we generated de Wit curves by varying the input of inoculated propagules of each strain to adjust strain frequency and by calculating either the numbers of colonies formed (colonization model alone) or the numbers of propagules produced (colonization and reproduction models coupled) as a function of the initial inoculum densities of the strains to measure RY. We also constructed curves using numbers of colonies of the strains for the input frequencies and calculating propagule production of each strain for the output yields (reproduction model alone). Calculations were conducted at three different total inoculum densities (Table 1). Model parameters were varied one at a time from their baseline values (Table 2) to determine how differences in each fitness component between strains affects the shape of de Wit curves. In many cases, the resulting de Wit curves were consistent with classical de Wit interpretations, but there were several notable exceptions that should be considered in using the de Wit analysis of interactions between competing organisms.

For the colonization model, the reproduction model, and the coupled colonization/reproduction models, we obtained the classical null lines for RY1, RY2, and RYT illustrated in Figure 1 when $E_{1}=E_{2}, K_{1}=K_{2}, A_{1}=A_{2}, M_{1}=M_{2}, \alpha_{1}=\alpha_{2}=1.0$, and $\beta_{1}=\beta_{2}=$ 1.0 , i.e., when all fitness parameters for the paired strains were equal and intrastrain interaction effects were identical to the interstrain interaction effects. For $\alpha_{1}=\alpha_{2}>1.0$ in the colonization

TABLE 1. Total inoculum densities used in model calculations to generate de Wit curves for two coexisting pathogen strains ${ }^{\mathrm{a}}$

\begin{tabular}{lc}
\hline Inoculum density & Value (propagules inoculated per leaf) \\
\hline Low & $1.0 \times 10^{2}$ \\
Intermediate & $2.0 \times 10^{4}$ \\
High & $2.0 \times 10^{5}$ \\
\hline
\end{tabular}

${ }^{a}$ Inoculum density was chosen based on data from the system of Puccinia graminis f. sp. tritici on wheat leaves.

b Densities were chosen to correspond to disease severities ranging from $<10 \%$ (low density) to $>90 \%$ (high density).

TABLE 2. Baseline values of fitness parameters used in model calculations to generate de Wit curves for two coexisting pathogen strains ${ }^{\mathrm{a}}$

\begin{tabular}{lll}
\hline Model & \multicolumn{1}{c}{ Parameter } & \multicolumn{1}{c}{ Value $^{\mathrm{b}}$} \\
\hline Colonization & Carrying capacity $(K)$ & $4.0 \times 10^{2}$ \\
& Infection efficiency $(E)$ & $5.0 \times 10^{-2}$ \\
\multirow{3}{*}{ Reproduction } & Interaction coefficient $(\alpha)$ & 1.0 \\
& Maximum propagule production $(M)$ & $2.0 \times 10^{5}$ \\
& Propagation efficiency $(A)$ & $4.0 \times 10^{3}$ \\
& Interaction coefficient $(\beta)$ & 1.0 \\
\hline
\end{tabular}

${ }^{a}$ Model parameter values were chosen based on data from the system of Puccinia graminis f. sp. tritici on wheat leaves.

$\mathrm{b}$ Baseline parameter values for all calculations. These values were varied in individual parameters at a time for specific comparisons. 
model and $\beta_{1}=\beta_{2}>1.0$ in the reproduction model (and all other parameters equal for the two strains), we obtained the classical concave downward de Wit curves expected for stronger interstrain than intrastrain competition (Fig. 2A). That this deviation below the null straight line is a general case for $\alpha>1.0$ can be seen from equation 2. The value of $U_{1}$ for $\alpha_{2}>1.0$ must be less than the value of $U_{1}$ for $\alpha_{2}=1.0$, because $x_{1} /\left(x_{1}+\alpha_{2} x_{2}\right)<x_{1} /\left(x_{1}+x_{2}\right)$ when $\alpha_{2}>1.0$. By the same reasoning, it can be seen from equation 4 that the value of $Q_{1}$ for $\beta_{2}>1.0$ must be less than the value of $Q_{1}$ for $\beta_{2}=1.0$. Also, we found that for $\alpha_{1}=\alpha_{2}<1.0$ and $\beta_{1}=\beta_{2}<1.0$, our colonization or reproduction models, respectively, yield the classical concave upward de Wit curves expected for stronger intrastrain than interstrain competition (Fig. 2B).

Contrary to classical expectations, however, we discovered that, under certain conditions, deviations from null straight lines for RY1 and RY2 could be produced even when intra- and interstrain competition were equal for both strains. This occurred when strains differed in infection efficiency $(E)$, regardless of whether yields were expressed in terms of colonies (colonization model alone) or propagules produced (coupled models). Such deviations also occurred when strains differed in carrying capacity for colony formation $(K)$, although only when yields were expressed in terms of propagule production (coupled models). Furthermore, we also found unexpected patterns of deviation from the null lines when strains differed in competitive ability for colony formation $(\alpha)$ and RYs were expressed in terms of propagule production (coupled models).

Colonization model alone. When strain 1 had an advantage in infection efficiency $(E)$ and all other traits were equal, output from the colonization model generated de Wit curves with RY1 above the expected null line and RY2 below the null line for equal interand intrastrain competition (Fig. 3). That is, the relative number of strain 1 colonies exceeded the de Wit expectation, and the number of strain 2 colonies was less than the de Wit expectation, given equal competitive abilities. These deviations from the null lines only become apparent at higher population densities, thus illustrating the criticism $(6,16)$ that the appearance of the curves, and there-

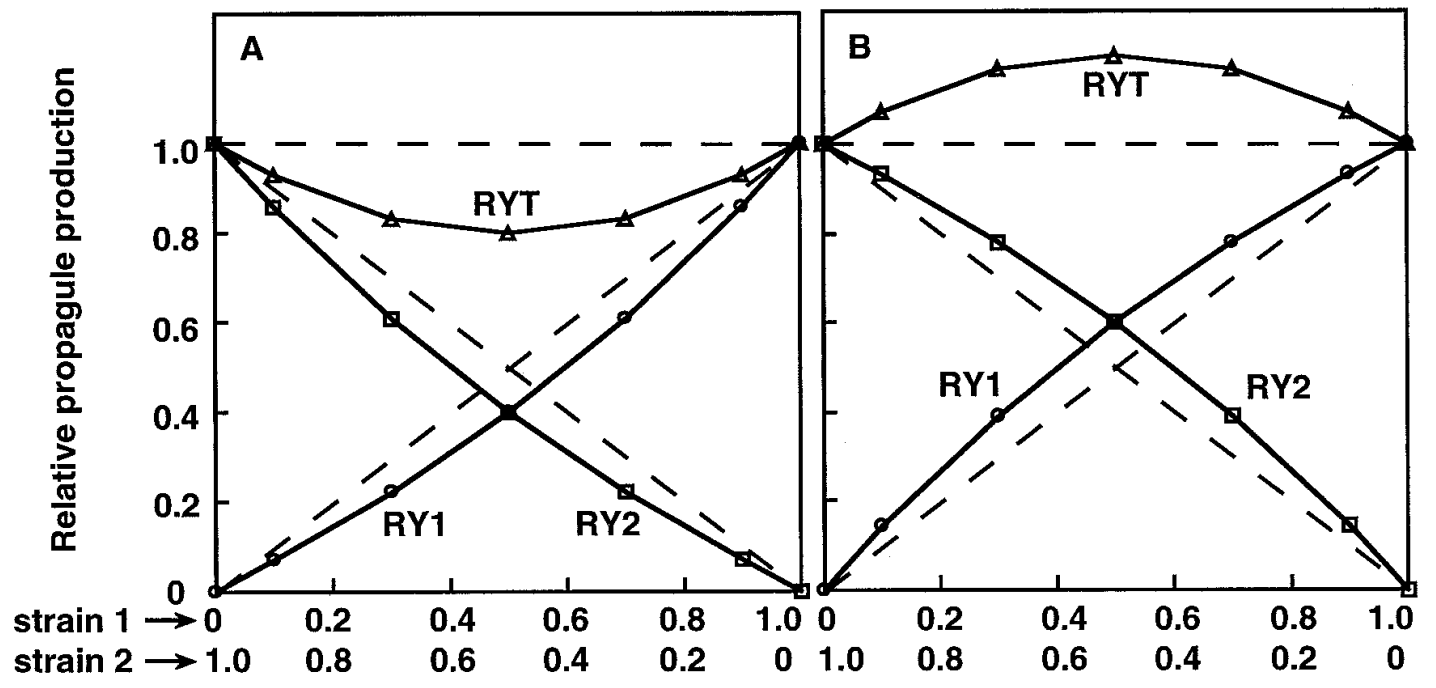

Proportion of strains in inoculum

Fig. 2. Classical de Wit curves of mutual inhibition or synergism between coexisting pathogen strains. Strains have equal baseline parameter values (Table 2), except that $\mathbf{A}$, interstrain competition > intrastrain competition for both strains $\left(\alpha_{1}=\alpha_{2}=1.5\right.$ in the colonization model and $\beta_{1}=\beta_{2}=1.5$ in the reproduction model); and $\mathbf{B}$, interstrain competition < intrastrain competition for both strains $\left(\alpha_{1}=\alpha_{2}=0.67\right.$ in the colonization model and $\beta_{1}=\beta_{2}=0.67$ in the reproduction model). High population-density calculations shown (Table 1). RY = relative yield and RYT $=$ RY total.

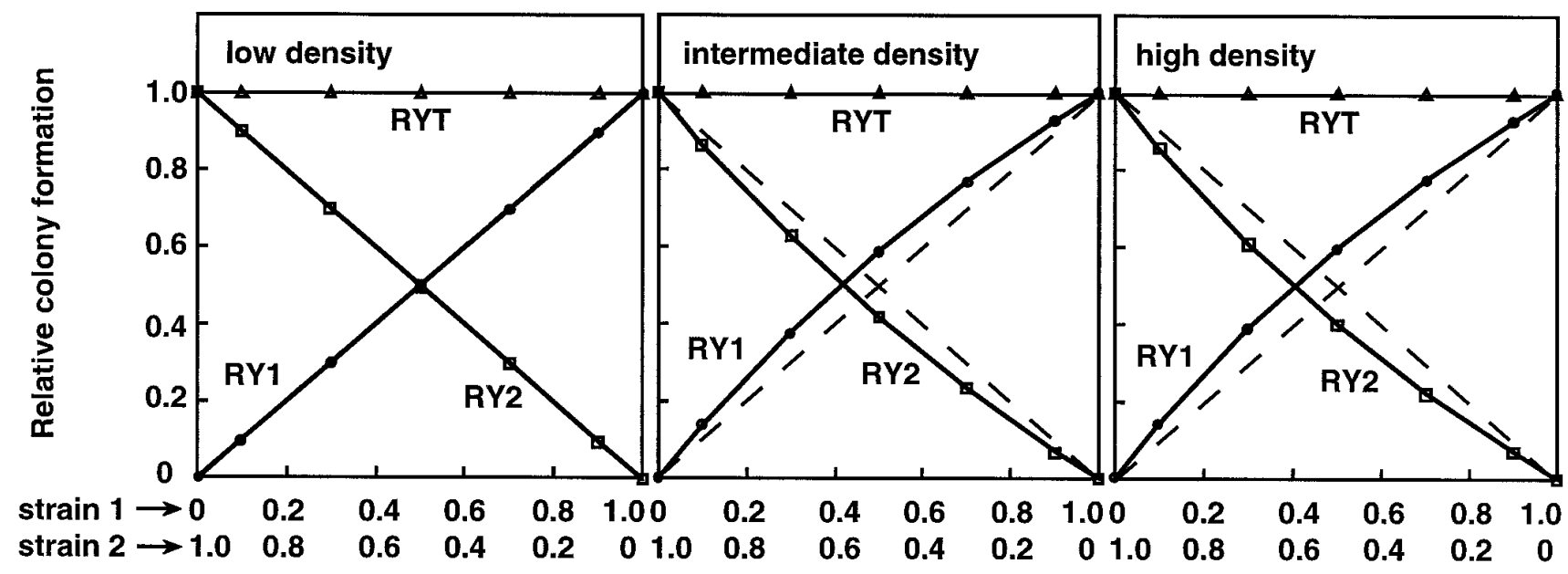

Proportion of strains in inoculum

Fig. 3. de Wit curves when two coexisting pathogen strains differ in infection efficiency (E): relative yield (RY) of each strain expressed in terms of colony formation on leaves. Strains have equal baseline parameter values (Table 2); except infection efficiency of strain 1 has been increased by $50 \%$. RYT $=\mathrm{RY}$ total. 
fore the interpretations that might be drawn from them, depend on population density. We found this same pattern of more obvious curvature with increasing population density in all de Wit plots constructed. In all other figures, we only present the high populationdensity results.

How is it possible for RYs of the paired strains to differ from their input frequencies in the mixture even though the inter- and intrastrain competitive abilities are exactly equal? In fact, the explanation is straightforward. When all other traits are equal in the paired strains, the strain with greater infection efficiency will be more successful in infecting localized infection sites, so its average frequency of infections per site will be greater than its input frequency in the inoculum mixture. Likewise, the strain with lower infection efficiency will achieve a lower frequency of infections per site than its initial frequency in the inoculum mixture. Since the strains are equal in their interaction abilities (interaction coefficients of both strains equal to 1.0), the probability that a strain will successfully colonize a multiply-infected site is equal to the proportion of infections of that strain in the site. Thus, the proportion of colonies formed by a strain with greater infection efficiency exceeds its original frequency in the inoculum mixture. Notice, however, that, in this case, $\mathrm{RYT}=1.0$ for all strain frequencies (Fig. 3), which is consistent with the de Wit expectation for equal inter- and intrastrain competitive abilities, even if the positions of the RY1 and RY2 curves are not.

When strains differed only in carrying capacity $(K)$, their relative colony yields coincided with the expected null lines (data not shown). A difference between strains in carrying capacity reflects a difference only in the number of sites that can support a successful colony of each strain and does not affect the relative numbers of infections produced by the strains in those sites. Because the strains in this case had equal infection efficiencies, the frequency of infections of a given strain per site available to that strain matched its frequency in the inoculum mixture, which resulted in that strain's frequency among established colonies in sites available to that strain also equaling its frequency in the inoculum. Thus, a difference between strains only in carrying capacity will result in relative colony yields that equal the initial frequencies of

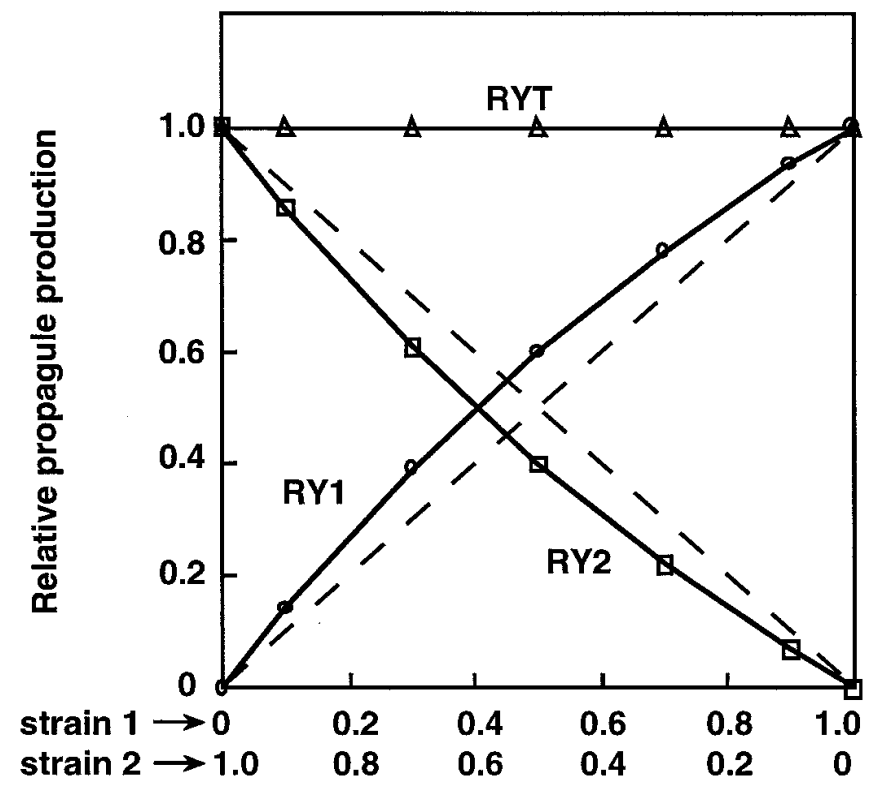

Proportion of strains in inoculum

Fig. 4. de Wit curves when two coexisting pathogen strains differ in infection efficiency $(E)$ : relative yield (RY) of each strain expressed in terms of propagule production on leaves. Strains have equal baseline parameter values (Table 2); except infection efficiency of strain 1 has been increased by $50 \%$. High population-density calculations shown (Table 1$)$. RYT = RY total. the strains in the inoculum, and thus, RY lines consistent with the null expectation.

When only the values of competitive ability for colony formation $(\alpha)$ were varied in the two strains, RY curves were consistent with traditional de Wit expectations. When the competition coefficient of either strain was less than, equal to, or greater than 1.0, the RY curve of the other strain was above, coincident with, or below its null line, respectively, as shown in Figure 2.

Reproduction model alone. For either $A_{1} \neq A_{2}$ or $M_{1} \neq M_{2}$ and all other parameters equal for the two strains with $\alpha_{1}=\alpha_{2}=1.0$ and $\beta_{1}=\beta_{2}=1.0$, the de Wit curves did not deviate from the null lines for RY1, RY2, and RYT, as shown in Figure 1. When the competition coefficients for propagule production $(\beta)$ in either strain were less than, equal to, or greater than 1.0, the RY curve of the other strain was above, coincident with, or below its null line, respectively, as shown in Figure 2. Thus, when numbers of colonies of the strains were used as the input frequencies and yields were expressed in terms of propagule production, results always corresponded to traditional de Wit expectations.

Colonization and reproduction models coupled. The de Wit curves for reproductive output generated from the coupled colonization and reproduction models were similar to those from the colonization model alone when strains differed only in infection efficiency $(E)$. The RY1 curve for strain 1 with an advantage in $E$ was above the null straight line, and the RY2 curve fell below the null line (Fig. 4). For strains differing only in colony-carrying capacity $(K)$, however, the de Wit curves from the coupled models differed from those from the colonization model alone. de Wit curves generated for the colonization model alone coincided with the null straight lines, but for the coupled model, the RY1 curve for strain 1 with an advantage in $K$ was above the null straight line and the RY2 curve was below it (Fig. 5). Thus, when strains differed only in carrying capacity for colonies, the strains' relative colony yields were as would be expected given the initial inoculum frequencies and outputs of the strains when alone, whereas the strains' propagule yields were not. Strain 1 with greater $K$ produced more propagules and strain 2 with lower $K$ produced fewer propagules than would have been predicted

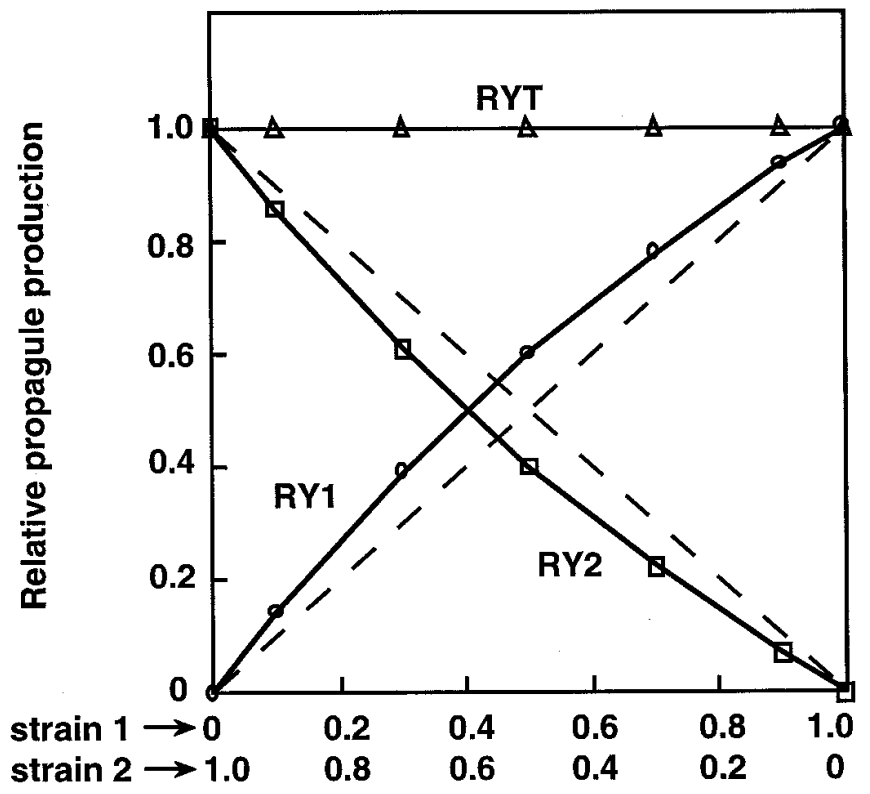

Proportion of strains in inoculum

Fig. 5. de Wit curves when two coexisting pathogen strains differ in carrying capacity for colonies $(K)$ : relative yield $(\mathrm{RY})$ of each strain expressed in terms of propagule production on leaves. Strains have equal baseline parameter values (Table 2), except strain 1's carrying capacity for colonies has been increased by $50 \%$. High population-density calculations shown (Table 1). RYT = RY total. 
based only on the strains' frequencies in the inoculum and their propagule production when alone.

When strains differed only in competitive ability for colony formation $(\alpha)$, the RY1 curve for strain 1 with an advantage in $\alpha$ was above its null line, the RY2 curve for the strain with the disadvantage fell below its null line, and the RYT curve coincided with the null line (Fig. 6). Figure 6 was generated using the values $\alpha_{1}=1.5$ and $\alpha_{2}=1.0$. According to traditional expectations (5), this combination of values should have resulted in a RY1 curve that coincided with the null line and RY2 and RYT curves below their null lines. However, we found that a difference between strains in $\alpha$ resulted in the same pattern as when strains differed in either one of the other infection model traits $(E$ or $K$ ) when yields were expressed in terms of propagule production.

The reason for such discrepancies from traditional de Wit expectations is that the coupled models represent two stages of interaction, the first in terms of colonies produced and the second in terms of propagules produced in the colonies. If all traits and intra- and interstrain competition are equal for two strains in the reproduction stage, the propagule production of a strain in mixture relative to its production alone at the same total density (RY) will approach its proportion in the colony population. Thus, differences between strains in traits at the colonization stage will result in different proportions in the colony population, and consequently, different RYs than would be traditionally expected based on initial inoculum frequencies.

\section{CONCLUSIONS}

Our results illustrate the importance of understanding the biology of the system in which competition is being analyzed. For most organisms, the relationships between initial populations and reproductive output, or populations at some subsequent point, are influenced by multiple fitness traits. For example, for rust fungi and many other fungal pathogens, reproduction involves four distinct steps: spore germination on the host, infection, survival and maturation of pathogen mycelia in lesions, and sporulation in the lesions.

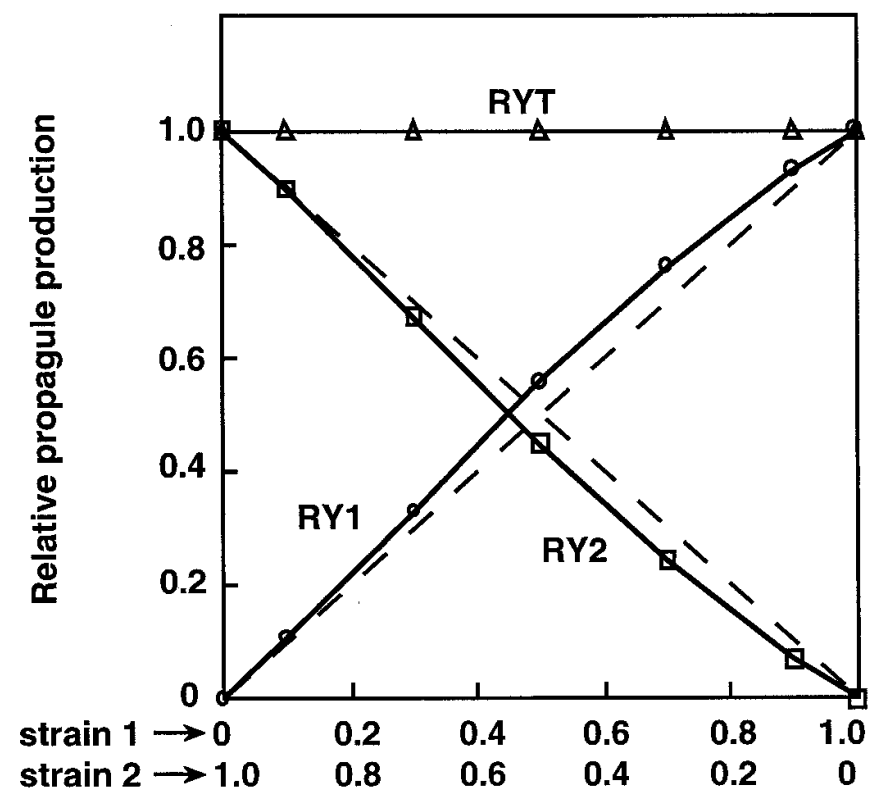

Proportion of strains in inoculum

Fig. 6. de Wit curves when two coexisting pathogen strains differ in competitive ability for colony formation $(\alpha)$ : relative yield (RY) of each strain expressed in terms of propagule production on leaves. Strains have equal baseline parameter values (Table 2), except strain 1's competitive ability for colony formation has been increased by $50 \%$. High population-density calculations shown (Table 1 ). RYT $=$ RY total.
Likewise, for pathogenic bacteria, infection-based reproductive output often requires preinfection growth, infection, and postinfection reproduction within lesions. Loss of individuals can occur at any stage. If the losses occur differentially for two coexisting strains, the proportions of the two strains can differ at different stages in the life cycle. de Wit analysis typically compares frequencies of competing strains at the beginning and at the end of a generation or time interval in which reproduction occurs. As we have shown, this can lead to misleading conclusions. For example, pathogen strains may differ significantly in infection efficiency $(E)$ and in the maximum numbers of reproductively active colonies or lesions $(K)$ that each can form on the plant surface $(9,12)$. Such differences can cause significant deviations of the RY1 and RY2 curves from the expected null straight lines in the de Wit analysis.

On the other hand, our results indicate that deviations from expected null RYT straight lines are produced only by differences between intra- and interstrain competition. Therefore, a significant deviation from the null RYT line is good evidence that intra- and interstrain competition differ significantly for at least one of the competing strains.

Absence of measurable deviation from the null RYT straight line, however, does not imply that intra- and interstrain competition are equal for both competing strains. Our results show that differences in intra- and interstrain competition during the colonization stage will not result in a deviation of the RYT curve from the null straight line for output of propagules from resulting colonies. Furthermore, all living organisms are complex and, with few exceptions, they differ from one another in many traits. Differences in parameters such as infection efficiency and carrying capacity for colonies per leaf as well as intra- and interstrain competition can produce complex patterns of interactions. For example, in the reproduction stage, one organism may exhibit greater interstrain competition with a second strain than intrastrain competition with itself. The reverse might be true for the second strain interacting with the first. A de Wit analysis of the interaction could produce an observed RYT curve that does not deviate significantly from the null straight line even though the observed RY1 and RY2 curves devi-

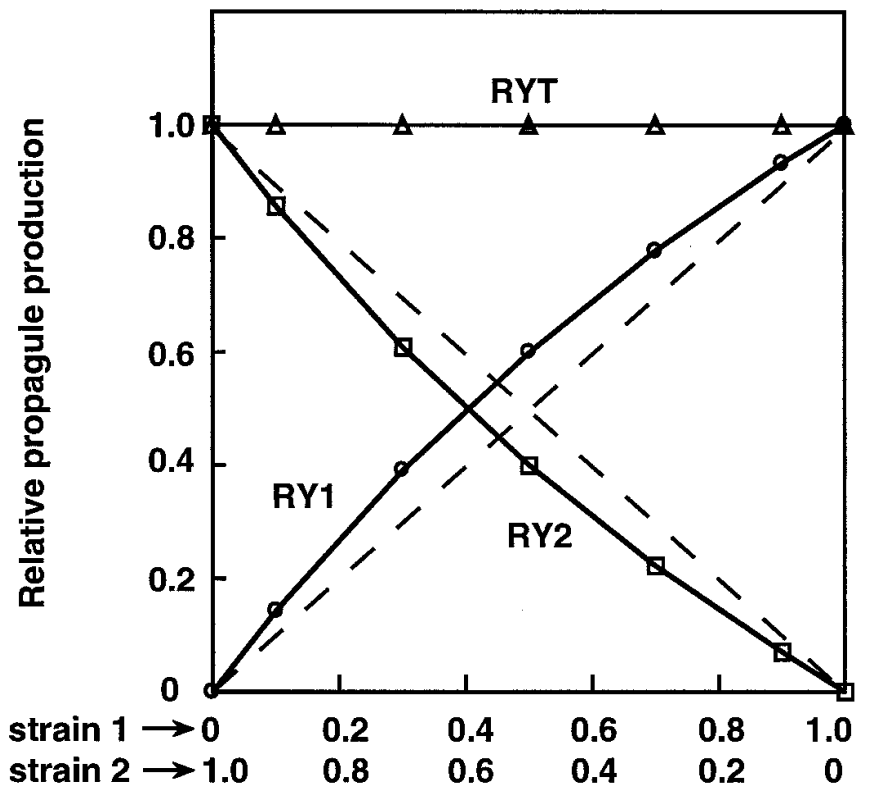

Proportion of strains in inoculum

Fig. 7. A straight relative yield total (RYT) line in de Wit curves does not necessarily indicate an absence of competitive differences between coexisting organisms. Strains have equal baseline parameter values (Table 2), except that strain 1 inhibits propagule production of strain 2 more strongly than strain 2 inhibits itself $\left(\beta_{1}=1.5\right)$, while strain 2 inhibits strain 1 less strongly than strain 1 inhibits itself $\left(\beta_{2}=0.67\right)$. High population-density calculations shown (Table 1). 
ated significantly from null straight lines (Fig. 7). Consider also that the two competing strains might differ in infection efficiency or carrying capacity for colonies per leaf, which would also cause deviations from the null straight lines for RY1 and RY2 without causing deviation of the observed RYT curve from the null line. Without further tests of the two strains individually, it would be impossible to know how much of the deviations from null RY1 and RY2 lines could be attributed to differences in infection efficiency or carrying capacity for colonies, and how much, if any, was due to differences in intra- and interstrain competition. The interactions could be even more complex if intra- and interstrain competition for the two strains were not only unequal, but also exhibited different inequalities in the colonization and reproduction stages.

From these considerations, we conclude that the results of de Wit analyses with pairs of plant pathogens or other similar microorganisms should be interpreted with caution. Deviations of the RYT curve from the null straight line are good evidence that intraand interstrain competition are unequal for either or both competing strains. However, without further experimentation, de Wit plots do not offer substantial insight into the mechanisms behind observed relative reproductive outputs in coexisting pathogen strains.

\section{ACKNOWLEDGMENTS}

We thank the Department of Plant Pathology at the University of Minnesota and the USDA, ARS Cereal Disease Laboratory for providing the resources for this work. The research was conducted under project $22-18 \mathrm{H}$ with funding from the department's H. H. Flor Fellowship awarded to M. Newton. We also thank J. Groth and R. Shaw for thoughtful comments that helped strengthen the manuscript. This manuscript was published as paper No. 0034 of the contribution series of the Minnesota Agricultural Experiment Station.

\section{LITERATURE CITED}

1. Adee, S. R., Pfender, W. F., and Hartnett, D. C. 1990. Competition between Pyrenophora tritici-repentis and Septoria nodorum in the wheat leaf as measured with de Wit replacement series. Phytopathology 80:1177-1182.

2. Antonovics, J., and Kareiva, P. 1988. Frequency-dependent selection and competition: Empirical approaches. Philos. Trans. R. Soc. Lond. B Biol. Sci. 319:601-613.

3. Campbell, C. L., and Madden, L. V. 1990. Introduction to Plant Disease Epidemiology. John Wiley \& Sons, New York.

4. da Luz, W. C., and Bergstrom, G. C. 1987. Interactions between Cochliobolus sativus and Pyrenophora tritici-repentis on wheat leaves. Phytopathology 77:1355-1360.

5. de Wit, C. T. 1960. On competition. Versl. Landbouwkd. Onderz. 66:1-82.

6. Firbank, L. G., and Watkinson, A. R. 1985. On the analysis of competition within two-species mixtures of plants. J. Appl. Ecol. 22:503-517.

7. Gregory, P. H. 1948. The multiple infection transformation. Ann. Appl. Biol. 35:412-417.

8. Johnson, N. L., and Kotz, S. 1969. Discrete Distributions. Houghton Mifflin, Boston.

9. Kinkel, L. L., Newton, M. R., and Leonard, K. J. 1996. Quantifying microbial competition on leaves. Pages 265-276 in: Aerial Plant Surface Microbiology. C. E. Morris, P. C. Nicot, and C. Ngyuen-the, eds. Plenum Press, New York,

10. Newton, M. R. 1996. Quantifying components of relative fitness in coexisting pathogen strains. M.S. thesis. University of Minnesota, St. Paul.

11. Newton, M. R., Kinkel, L. L., and Leonard, K. J. 1996. Density- and frequency-dependent fitness in coexisting foliar pathogens. Phytopathology 86:S85.

12. Newton, M. R., Kinkel, L. L., and Leonard, K. J. 1997. Competition and density-dependent fitness in a plant parasitic fungus. Ecology 78:1774-1784.

13. Newton, M. R., Kinkel, L. L., and Leonard, K. J. 1998. Determinants of density- and frequency-dependent fitness in competing plant pathogens. Phytopathology 88:45-51.

14. Rai, B., and Upadhyay, R. S. 1983. Competitive saprophytic colonisation of pigeon-pea substrate by Fusarium udum in relation to environmental factors, chemical treatments and microbial antagonism. Soil Biol. Biochem. 15:187-191.

15. Roughgarden, J. 1979. Theory of Population Genetics and Evolutionary Ecology: An Introduction. Macmillan, New York.

16. Snaydon, R. W. 1991. Replacement or additive designs for competition studies? J. Appl. Ecol. 28:930-946.

17. Widden, P. 1984. The effects of temperature on competition for spruce needles among sympatric species of Trichoderma. Mycologia 76:873-883.

18. Widden, P., and Hsu, D. 1987. Competition between Trichoderma species: Effects of temperature and litter type. Soil Biol. Biochem. 19:89-93

19. Wilson, M., and Lindow, S. E. 1995. Enhanced epiphytic coexistence of near-isogenic salicylate-catabolizing and non-salicylate-catabolizing Pseudomonas putida strains after exogenous salicylate application. Appl. Environ. Microbiol. 61:1073-1076. 